\title{
Mobile-Based Synchronization Model for Presentation of Multimedia Objects
}

\author{
Keun-Wang Lee ${ }^{1}$, Hyeon-Seob $\mathrm{Cho}^{2}$, and Kwang-Hyung Lee ${ }^{3}$ \\ 1 Dept. of Multimedia Science Chungwoon University, Korea \\ kwlee@cwunet.ac.kr \\ 2 Dept. of Electronics Engineering Chungwoon University, Korea \\ ${ }^{3}$ School of Computing, Soong-sil University, Korea
}

\begin{abstract}
This paper presents a synchronization model that implements the presentation of multimedia objects as well as a synchronization scheme that improves media data latency and quality of service (QoS) in mobile environments. The proposed model meets synchronization requirements among multimedia data because it employs an approach to not only adjusting synchronization intervals using the maximum available delay variation or jitter at the base station (BS) but also flexibly dealing with variable latencies due to variations in delay time.
\end{abstract}

\section{Introduction}

Synchronization is a critical prerequisite to ensuring an adequate QoS in providing multimedia services. The reason behind that lies in the disruption of original timing relations resulting from the occurrence of random delays in mobile networks or mobile host $(\mathrm{MH})$ system clock inconsistencies among inter-media, all of which is attributable to difference in the time of arrival of data being transferred from the server side to the $\mathrm{MH}$ over wireless communications networks. Therefore, for the multimedia data in which timing relations are disrupted, an artificially synchronized presentation of data streams is required to ensure similarity or identicalness to the original ones, through the use of the requirements for application services or the limitations of human perception toward loss and delays occurring in individual media [1][2][3].

This paper deals with live synchronization involving the real-time synchronization of multimedia information as well as synthetic synchronization for stored media. For this example, let us assume that we are taking 3D animation training for electrical safety from a MH. While a lecturer is providing verbal explanations, his/her voice, moving images, texts, and 3D animation should be presented simultaneously. As in wired environments, smooth audio/visual presentations should be delivered in mobile environments. In this regard, this paper proposes a synchronization model that allows for simultaneous presentations of multimedia objects such as voice, 3D animation, moving images and texts on a $\mathrm{MH}$ as well as presents an intra-media and inter-media synchronization scheme that enables mobile multimedia services.

\section{Related Work}

Previous studies have focused on describing synchronization models for multimedia applications [5][6]. Among them, Petri-net based specification models are effective in 
specifying temporal relationships among media objects. These models allow various media to be integrated and give easy descriptions of QoS requirements. However, previous extended forms of Petri-net modeling like Object Composition Petri-Net (OCPN) and Real Time Synchronization Model (RTSM) exhibit limitations as far as QoS parametric modeling is concerned.

Many existing studies pose significant problems in that delay in wireless networks causes a reduction in the maximum media playout latency. That's because conventional research places focus on determining the sequence and playout time of the media created by the server side and fixing the queuing time at the buffer on the MH side.

Delay jitter causes discontinuity. If any discontinuity is permissible at a $\mathrm{MH}$, it should be smaller than the worst end-to-end playout delay. This case leaves you with two strategy options; one with I-strategy in which belatedly arrived frames from the BS are discarded, and the other with E-strategy in which belatedly arrived frames from the BS are played out.

This paper proposes a dynamic synchronization scheme that adjusts intervals between synchronization activity using the maximum available delay variation or jitter, reduces data loss caused by variations in latency, and synchronizes inter-media and intra-media.

\section{Proposed Synchronization Model}

The proposed synchronization model is Petri-net based standard model that enables the presentation of multimedia objects.

\subsection{Definition of Synchronization Model}

The proposed synchronization model for specifying Petri-net in any BS is defined as follows:

The model is specified by the tuple $[P, T, K, A, R e, M]$

where

$P=p_{1}, p_{2}, \ldots, p_{n} ;$ Regular places(single circles).

$T=t_{1}, T_{2}, \ldots, t_{m} ;$ Transitions.

$K=k_{1}, k_{2}, \ldots, k_{i} ;$ Key places.

$X=P \cup K ;$ All places.

$A=(X \times T) \cup(T \times X) \rightarrow I, I,=1,2,3, \ldots ;$ Directed arcs.

Re $: X \rightarrow r_{1}, r_{2}, \ldots, r_{k}$; Type of media.

$M: X \rightarrow I, I=0,1,2 ;$ State of places

The "Place" is used to represent a medium unit and its action. It may have tokens. A place without any token indicates that it is currently in an inactive state. A place with a token stands in an active state and may be in either a blocked or unblocked state.

The information determining the firing of a transition $t_{i}$ is delivered by a control medium. When a key medium arrives within the time specified by an absolute time, the transition $t_{i}$ is immediately fired at the corresponding transition's input place that has open tokens. However, in the event that the key medium reaches beyond the time specified by an absolute time, the absolute time initiates the firing. 


\subsection{Control Medium}

As a control medium, CT has the information about the number of input places and key media while transmitting this information to a subsequent transition. The roles of a control medium is addressed by the following:

(1) Check the set of input places;

(2) Determine the number of objects selected as key media

(3) Transmit the number of input places and key media as well as an absolute time to a subsequent transition.

Figure 1 shows the state of a key medium and the active state stored in the control medium, which will be transmitted to subsequent transitions. 7 bits at the front and 7 bits at the back indicate the information of a key medium and the information of its active state, respectively.

\begin{tabular}{|l|c|c|c|c|c|c|c|c|c|c|c|c|c|}
\multicolumn{10}{c|}{ Key Medium state } & \multicolumn{10}{c|}{ active state } \\
\hline K_T & Au & I & Ani & Tx & Po & V & a_T & Au & I & Ani & Tx & Po & V \\
\hline
\end{tabular}

Fig. 1. Information of Control Medium

All media are transmitted over networks, so real-time constraints are likely to be exceeded. In the event of the delay of a key medium, a firing is available via the absolute time of the control medium.

\subsection{Presentation of Synchronization Model}

Figure 2 shows an overall view of Petri-nets. 1'(125,3,1,1,1,0,0,0,6,1,1,1,1,1,1) indicates the information of a control medium. The first parameter 125 refers to the absolute time, 3 the number of key media, and 6 the number of input places. As Control indicates type, Control_in is applicable to all conditions. When an event (i.e. number of key media and number of input places) occurs, the control medium transmits the information to subsequent transitions.

HS indicates a hierarchical submodule that goes to a lower level module for job processing before going to the next phase. HS goes to a relative duration routine - one of its lower level modules - to find the relative duration before executing After_duration. Therefore, HS has synchronization intervals three times, which results in effective synchronizations in a way that computes the relative duration before executing syncl, computes the compensation time for jitters before executing sync2, and computes the flexible playout time before executing sync3.

Verifications of the proposed multimedia synchronization model have been performed in order to make sure that it corresponds to the analysis methods of petri-nets including a reachability graph and a matrix-equation.

Verification of Reachability Graph. The reachability tree represents the reachability set of petri-nets. In the proposed model, the initial marking is $(1,1,1,1,1$, 


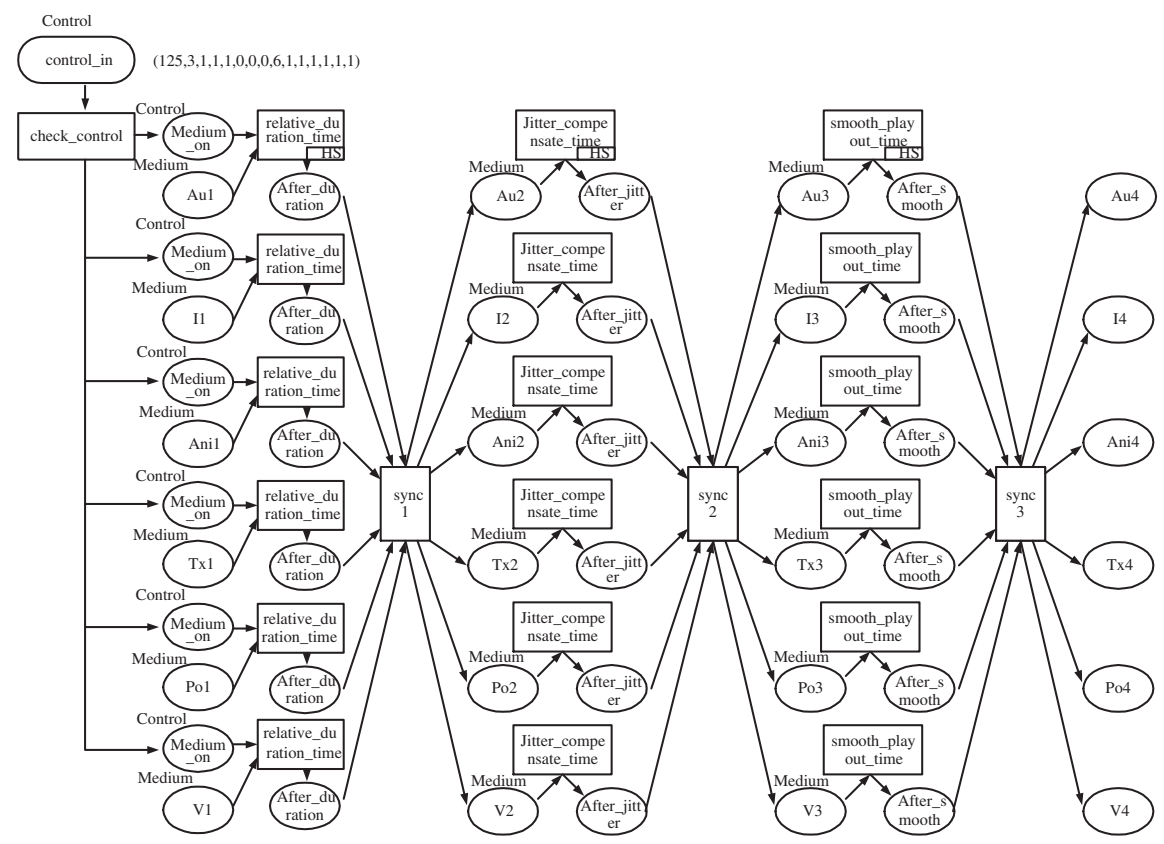

Fig. 2. Presentation of Synchronization Model

$1,0,0,0,0,0,0,0,0,0,0,0,0)$. The transition $t_{1}$ is generated from this initial marking. If $t_{1}$ is fired, $t_{1}$ [giving $(0,0,0,0,0,0,1,1,1,1,1,1,0,0,0,0,0,0)$ ] is obtainable, and a transition to $t_{2}$ is possible. If $t_{2}$ is fired, $t_{2}$ [giving $(0,0,0,0,0,0,0$, $0,0,0,0,0,1,1,1,1,1,1)]$ is obtainable. The result of this tree is represented as follows:

$$
\begin{aligned}
(1,1,1,1,1,1,0,0,0,0,0,0,0,0,0,0,0,0) \\
\\
\quad \downarrow t_{1} \\
(0,0,0,0,0,0,1,1,1,1,1,1,0,0,0,0,0,0) \\
\quad \downarrow t_{2} \\
(0,0,0,0,0,0,0,0,0,0,0,0,1,1,1,1,1,1)
\end{aligned}
$$

Verification of Matrix-Equation. Verifications of the proposed model were performed using matrix-equations as the second analysis method of the petri-nets. The following are two matrices $D^{-}$and $D^{+}$indicating input function and output function, respectively. Matrices $D^{-}$and $D^{+}$are expressed as follows:

$$
\begin{aligned}
D^{-} & =\left[\begin{array}{l}
1,1,1,1,1,1,0,0,0,0,0,0,0,0,0,0,0,0 \\
0,0,0,0,0,0,1,1,1,1,1,1,0,0,0,0,0,0
\end{array}\right] \\
D^{+}= & {\left[\begin{array}{l}
0,0,0,0,0,0,1,1,1,1,1,1,0,0,0,0,0,0 \\
0,0,0,0,0,0,0,0,0,0,0,0,1,1,1,1,1,1
\end{array}\right] }
\end{aligned}
$$

And the matrix D is expressed as follows: 


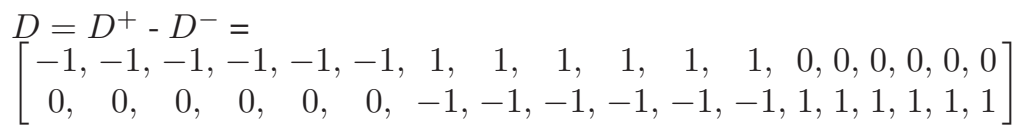

The result of applying the equation $\mu^{\prime}=\mu+x \cdot D$

to the matrices is addressed by the following :

$(0,0,0,0,0,0,0,0,0,0,0,0,1,1,1,1,1,1)=$

$(1,1,1,1,1,1,0,0,0,0,0,0,0,0,0,0,0,0)$

$x\left[\begin{array}{ccc}-1,-1,-1,-1,-1,-1,1, \quad 1, \quad 1, \quad 1, \quad 1, \quad 1,0,0,0,0,0,0 \\ 0, \quad 0, \quad 0, \quad 0, \quad 0, \quad 0,-1,-1,-1,-1,-1,-1,1,1,1,1,1,1\end{array}\right]$

$(-1,-1,-1,-1,-1,-1,0,0,0,0,0,0,1,1,1,1,1,1)=$

$X\left[\begin{array}{ccc}-1,-1,-1,-1,-1,-1,1, \quad 1, \quad 1, \quad 1, \quad 1, \quad 1,0,0,0,0,0,0 \\ 0, \quad 0, \quad 0, \quad 0, \quad 0, \quad 0,-1,-1,-1,-1,-1,-1,1,1,1,1,1,1\end{array}\right]$

where $\mathrm{x}$ is $\left(x_{1}, x_{2}\right)$

$-1=-1 \cdot x_{1}$

$0=x_{1}-x_{2}$

$1=x_{2}$

$1=x_{2}$

For (1), $x_{1}=1$;

For (2), $x_{1}=1$;

Therefore, for (3), $x_{1}-x_{2}=0$

Through the verification described above, the proposed synchronization model proved to be consistent with the reachability graph and matrix-equation.

\section{Synchronization Scheme}

This chapter describes a delay jitter scheme. Delay time or latency is an important factor in evaluating the playout quality of a frame. This paper provides a description of delay jitter strategies such as I-strategy and E-strategy. It also makes a comparison between those two strategies and the proposed delay jitter strategy using the waiting time in the queue as well as playout time.

[Theorem] A frame waits for as long as variable latency or jitter times until they are presented.

[Proof] Where the maximum latency or jitter time is below $10 \mathrm{~ms}$, temporary discontinuity is tolerable for voice media without causing any impact to quality of service. If the discontinuity tolerance is $\delta$, an extended synchronization interval becomes $\Delta^{\prime}=\Delta^{\prime}$ 
$+\delta$. Therefore, if the instantaneous discontinuity tolerance permitted by the corresponding media data is $\delta$, an extended synchronization interval becomes $\Delta^{\prime}=\Delta+\delta$. If the $(j+1)$ th packet arrival time at the buffer is $B_{i(j \cdot i)}$, the synchronization requirements are met when $B_{i(j \cdot 1)}$ is smaller than or equal to the frame playout time $M_{i(j \cdot i)}$. Formula 1 meets the synchronization requirements.

$B_{i j}<M_{i(j \cdot 1)}$

$B_{i j}<M_{i j}+\Delta^{\prime}$

$B_{i j}<M_{i j}+\Delta+\delta$

$B_{i j}<M_{i j}+1 / N+\delta \cdots($ Formula 1$)$

The proposed strategy illustrates that, if the $(j)$ th packet for media data stream $i$ is presented at the time $M_{i j}$, the $(j+1)$ th packet is presented at the time $M_{i(j+1)}=M_{i j}+$ $\Delta^{\prime}$. In other words, $(j)$ th and $(j+1)$ th packets meet Formula 1 within synchronization intervals.

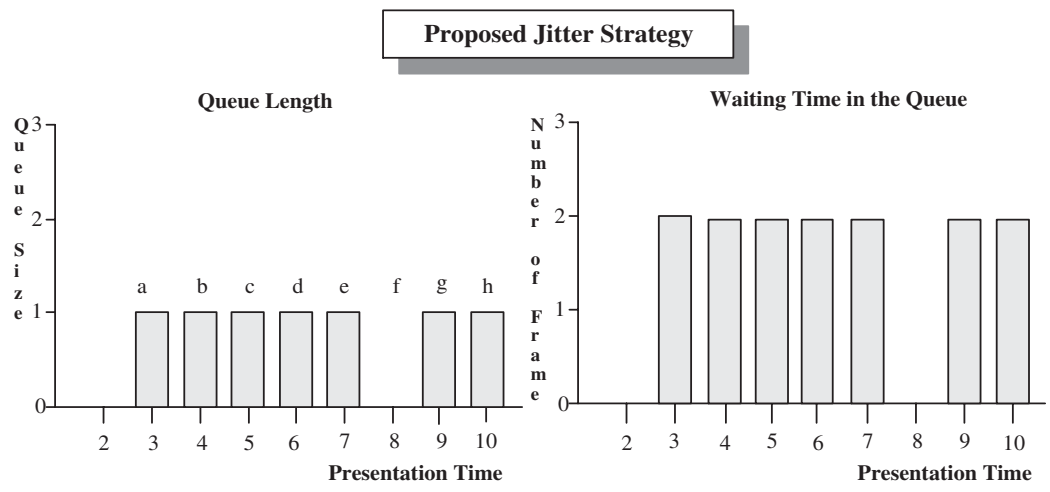

Fig. 3. Proposed Strategy Applied Under Reduced Network Traffic

Figure 3 shows the proposed jitter strategy that complements the shortcomings of both the I-strategy and E-strategy. Belatedly arrived frames wait for as long as latency or jitter times until they are presented, instead of being deleted unconditionally or waiting indefinitely until they are presented at the next playout time. As depicted in Figure 3, the frames $b$ and $c$ are played out in the units 4 and 6 by compensating for the maximum latency or jitter. The frame $f$ indicates that it has not arrived within the variable delay jitter time. In this case, the frame $f$ cannot be played out even if it waits for as long as the variable latency or jitter times.

Therefore, the unit 8 in the frame $f$ indicates that it cannot be compensated for the maximum delay jitter time due to excessive delay.

Figure 4 shows that the unit 4 in the frame $c$ was skipped due to excessive delay and the unit 5 in the frame $d$ was compensated by applying the maximum delay jitter time. 


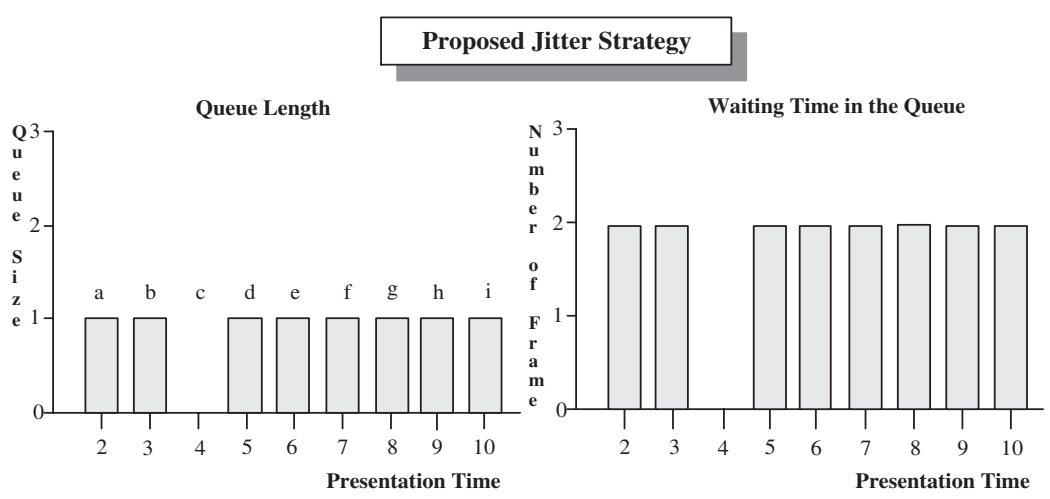

Fig. 4. Proposed Strategy Applied Under Heavy Network Congestion

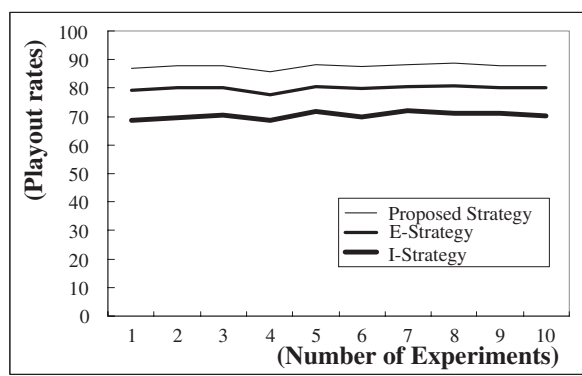

Fig. 5. Comparison of Playout Rates

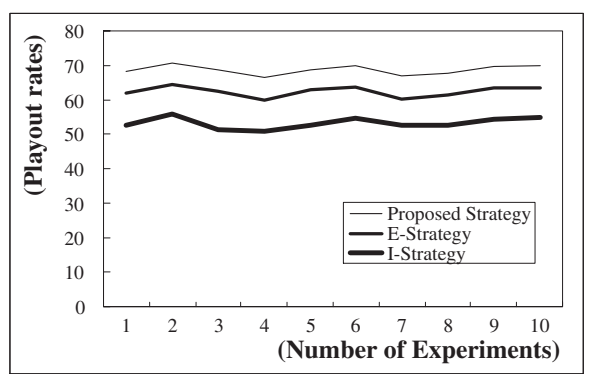

Fig. 6. Comparison of Playout Rates

\section{Performance Evaluation}

With focus on the playout time and loss time, we performed a comparative analysis between the existing scheme and the delay jitter \& playout scheme using the maximum delay jitter time. Comparisons among the I-strategy, E-strategy, and the proposed strategy were made in consideration of the following two cases; Circumstances of reduced network traffic and circumstances of heavy network congestion. As a result, the proposed strategy proved to be vastly superior to other two strategies. For the purpose of this paper, we have assumed that the average delay is $100 \mathrm{~ms}$ and the variance is $20 \mathrm{~ms}$ in the event of delay in the audio stream.

Figure 5 shows the result of comparisons of playout rates among the I-strategy, Estrategy, and the proposed strategy that were applied under reduced network traffic. The playout rates of the proposed strategy were obtained by conducting experiments ten times. The proposed strategy showed more improved playout rates than the I-strategy and E-strategy by $17.33 \%$ and $7.8 \%$, respectively.

As shown in Figure 6, under heavy network congestion, the proposed strategy showed more improved playout rates than the I-strategy and E-strategy by $15.48 \%$ and $6.3 \%$, respectively. 


\section{Conclusions}

This paper has proposed a scheme for implementing intra-media and inter-media synchronizations by means of smooth buffering at the BS in mobile environments. The proposed scheme delivered optimized synchronizations without causing any degradation of quality of service. The superiority of the proposed scheme was demonstrated by extending intra-media synchronization intervals using the maximum delay jitter time of the audio media as a key medium, as well as by synchronizing irregularly arriving packets within the extended intervals through applications of the said maximum delay jitter time to inter-media synchronizations.

The proposed scheme for enabling intra-media and inter-media synchronizations is ideally suitable for the temporary increase in network load as well as unforeseeable disconnections.

Furthermore, it allows us to take 3D animation training for electrical safety while on the road.

Future work needs to focus not only on standard schemes for mobile multimedia synchronizations which take interactions with users into account, but also on optimized synchronization mechanisms which employ minimum buffering.

Acknowledgements. This research is supported by the Electric Power Industry R\&D Fund 2003 supported by Ministry of Commerce, Industry and Energy in republic of Korea.

\section{References}

1. D. H. Nam and S. K. Park, "Adaptive Multimedia Stream Presentation in Mobile Computing Environment," Proceedings of IEEE TENCON, 1999.

2. A. Boukerche, S. Hong and T. Jacob, "MoSync: A Synchronization Scheme for Cellular Wireless and Mobile Multimedia Systems," Proceedings of the Ninth International Symposium on Modeling, Analysis and Simulation of Computer and Telecommunication Systems IEEE, 2001.

3. M. Woo, N. Prabhu and A. Grafoor, "Dynamic Resource Allocation for Multimedia Services in Mobile Communication Environments,"IEEE J. selected Areas in Communications, Vol.13, No.5, June. 1995.

4. D. H. Nam, S. K. Park, "A Smooth Playback Mechanism of Media Streams in Mobile Computing Environment," ITC-CSCC'98, 1998.

5. P. W. Jardetzky, and C. J. Sreenan, and R. M. Needham, "Storage and synchronization for distributed continuous media," Multimedia Systems/Springer-Verlag, 1995.

6. C.-C. Yang and J.-H. Huang, "A Multimedia Synchronization Model and Its Implementation in Transport Protocols," IEEE J. selected Areas in Communications, Vol.14, No.1, Jan. 1996. 